Research Article

Gianfranco Piana*, Valerio De Biagi, and Bernardino Chiaia

\title{
Robustness of an airport double layer space truss roof $^{\star *}$
}

https://doi.org/10.1515/cls-2021-0004

Received Jul 30, 2020; accepted Jan 01, 2021

\begin{abstract}
Robustness analyses are very well referenced for concrete or steel frame structures but less for spatial structures; in particular for truss roofs. Here, we present a robustness analysis of an existing airport space structure. A finite element model was implemented based on the original design documents, where the structure is modeled as a spatial truss composed of elastic, perfectly hinged bars. With respect to five main design loading conditions, the most stressed bars among the top layer, the bottom layer and the diagonals, were alternately removed, and the stress variations in the remaining bars monitored. A total of fifteen analyses with removal of either the most stretched or the most compressed bar were run. Also, reductions of the most stressed bars' axial stiffness were considered to investigate the effects of such local reductions on the global structural flexibility. Linearized global buckling analyses were also conducted to point out again the effect of a global loss of stiffness, due to local losses. The study gives basic information about the general behavior of the structure in case of failure, or damage, of a key element. Results show that this kind of lightweight and efficient structures are very sensitive to local losses, since their redistribution capabilities are not large.
\end{abstract}

Keywords: space frame structure, airport roof, structural robustness, extreme event, damage

\footnotetext{
*Corresponding Author: Gianfranco Piana: Department of Struc-
tural, Geotechnical and Building Engineering, Politecnico di Torino,

*Corresponding Author: Gianfranco Piana: Department of Struc-
tural, Geotechnical and Building Engineering, Politecnico di Torino, Corso Duca degli Abruzzi 24, 10129 Torino, Italy; Department of Bridge Engineering, Tongji University, 1239 Siping Road, Shanghai, China; Email: gianfranco.piana@polito.it

Valerio De Biagi, Bernardino Chiaia: Department of Structural, Geotechnical and Building Engineering, Politecnico di Torino, Corso Duca degli Abruzzi 24, 10129 Torino, Italy

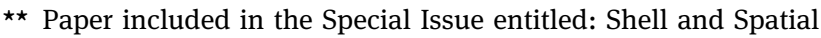
Structures: Between New Developments and Historical Aspects
} əopen Access. ( 2021 G. Piana et al., published by De Gruyter. (cc) BY License

\section{Introduction}

Space truss roofs are lightweight, convenient and economic solutions to cover large areas. Their structural efficiency makes them working with low safety margins even with respect to regular loading conditions. At the same time, their public function requires a maintenance plan including structural health inspections and checks for robustness against extreme events, like fire, explosion, accidental failure of an element, etc. [1]. When subjected to a local failure, a robust structure is able to redistribute the forces in the remaining members [2]. As reported in the Eurocode 0 [3], in a robust structure "consequences of structural failure should not be disproportional to the effect causing the failure". Robustness studies have mainly focused on frames (e.g., [4]) and slab-column structural types [5], as they are the most diffused types of resisting structures. The results of such researches have recently merged into design guidelines. On the contrary, very limited literature focuses on the collapse and the robustness of large span spatial grid structures [6]. The early studies on element removal on double layer gridshell structures date back to the Nineties with the work by Hanaor [7], who assessed the residual capacity of damaged roofs of variable size as soon as the critical elements in compression or in tension were removed. In addition, he observed on real scale experiments that the high degree of static redundancy, rather than providing "positive" effects and force redistribution, lead to a reduction of the safety margin since the system is too much sensitive to imperfections. The importance of details in the design has been highlighted after the forensic studies for understanding the causes of a gym roof failure in North Italy [8]. The importance of a precise erection technique, of a good detailing of the connections, of the nodal imperfections, have been proved by the various collapses that occurred in long-span grid structures, as reported in [9]. In general, it can be stated that loss of tension bars can happen due to bolt/node failure, while loss of compression bars could happen due to flexural buckling induced by overloading or differential settlements of the supports [10], if global ductility is not large enough [7]. 
Referring to the robustness of such long-span structures, the studies mainly focused on single layer grids. In general, element removal and simulation of the behavior of the roof were performed to respond to some design questions: optimize the shape of the structure to resist progressive collapse robustness $[11,12]$ or monitoring the damaged structure [13].

Here, we present a robustness analysis of an airport double layer space frame structure. The structure is existing; according to the owner's wish, we will keep the name confidential. A finite element model was implemented which reflects geometry, material and section properties, constraints and loads as specified in the original design documents. As the designers did, the structure was modeled as a spatial truss composed of perfectly hinged elastic bars. Among a total of 65 , five main loading conditions were extracted from the original structural design report, which include the following actions: self-weight, other permanent loads, snow and temperature gradients. Among all, the seismic load combination was not selected and included in the present study because it represents a condition less severe than the five considered here, since the building falls in a low-seismicity area. For each of the selected loading conditions, the most stretched bar and the most compressed bar among the top layer, the bottom layer and the diagonals, and the most compressed bar among the support diagonals, were alternately removed, and the stress variations in the remaining bars monitored. A total of fifteen analyses with removal of the most stretched or the most compressed bar were run. Also, reductions of the most stressed bars' axial stiffness (i.e., cross-sectional area) were considered to compare the single contributions to the global structural flexibility. Bar removals can be brought back to tensile failure or to compressive buckling, whereas bar stiffness reductions can be associated to a damage due, for example, to corrosion. Lastly, linearized global buckling analyses were also performed to further investigate the effect of a global loss of stiffness, due to local removals.

\section{Methods}

A numerical model of the roof was implemented in LUSAS finite element code. Such model reflects geometry, material and section properties, constraints and loads as specified in the original structural report. The geometry has been inferred from the structural drawings. The structure was modeled as a space truss made of perfectly hinged, elastic bars. The model is composed of 796 nodes and 3013 bars (Figure 1). The global geometry, although made up by a regular scheme, results complex due the huge dimensions and the very large number of elements. Any complete 3D view would result unclear. Therefore, here we will only give a sketch of the real roof, mainly aiming at describing the essentials of the structural scheme.

The roof has maximum plan dimensions of $(86.4 \times 57.6)$ $\mathrm{m}$ and presents a small curvature in the transverse (shorter) direction (Figures 1a, 1b). The centers of curvature are fixed and the radii of curvature are equal to about $693.66 \mathrm{~m}$ for the top layer and $690.46 \mathrm{~m}$ for the bottom layer. The top layer grid has dimensions $(3.6 \times 3.614) \mathrm{m}$, while the bottom layer one has dimensions $(3.6 \times 3.597) \mathrm{m}$. The distance between the two layers is $3.2 \mathrm{~m}$. Global axes X, Y, Z in Figure 1 help interpreting the geometry.

The roof is constrained in the vertical direction at 38 nodes, 34 of which are along the side dish and 4 are the in the central part; the perimeter constraints are able to react only in the upward direction, while the central ones are bilateral constraints (Figures 1 and 2). In the horizontal direction, the structure is constrained at 3 of the 4 central supports so to furnish two reaction points for each of two main directions (Figure $2 \mathrm{~b}$ ). Figure $2 \mathrm{c}$ shows a bottom view around a central support. Figures $2 \mathrm{~d}$ and $2 \mathrm{e}$ show a detail picture around a node and the corresponding scheme, respectively. In Figure 2e, indicated in black are a node of the bottom layer, the four diagonal bars connecting it to the top layer, and the visible part of the top layer.

The bars, made of S355 steel (Young's modulus $E=$ $210 \times 10^{3} \mathrm{MPa}$, yield stress $f_{y k}=355 \mathrm{MPa}$, tensile strength $f_{t k}=510 \mathrm{MPa}$ ), have circular hollow sections with the eight

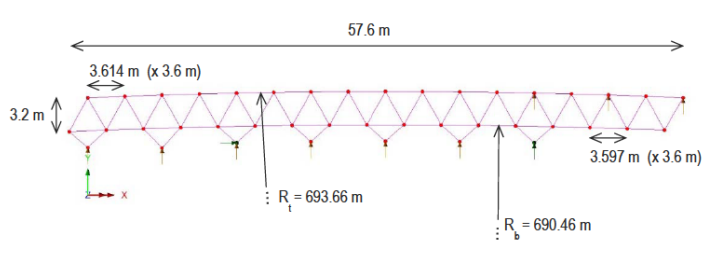

(a)

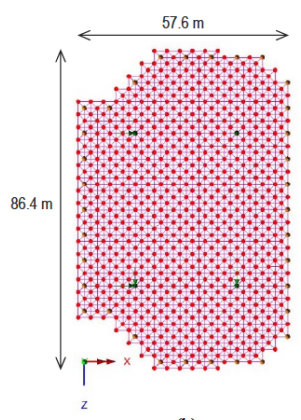

(b)

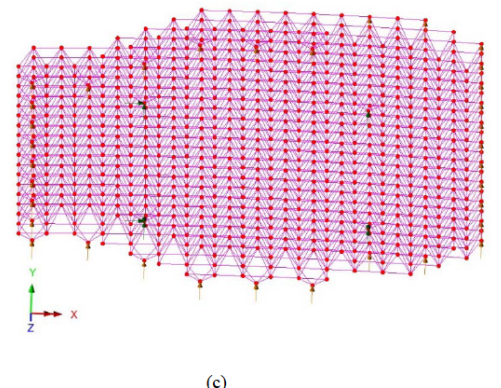

(c)
Figure 1: Finite element model of the analyzed space truss roof, with main dimensions indicated (not to scale): (a) side view (XY-plane), (b) top view (XZ-plane), and (c) axonometric view 


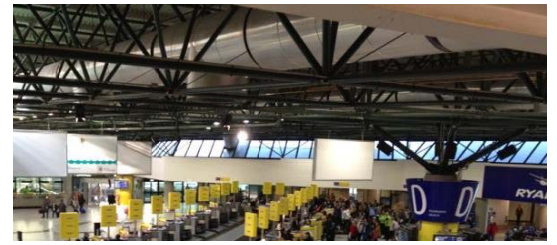

(a)

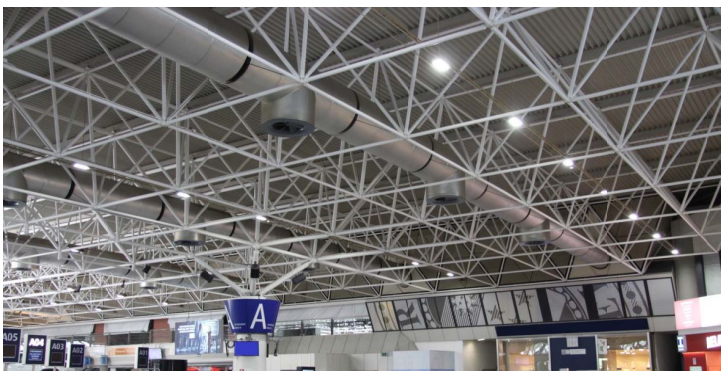

(c)

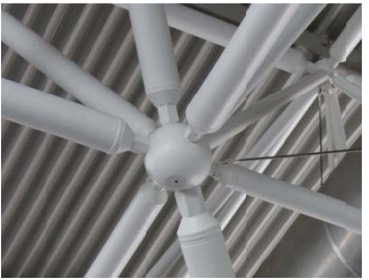

(d)

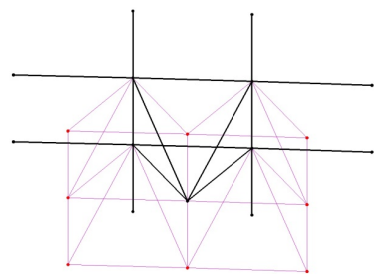

(e)
Figure 2: (a) Bottom view of the truss roof with central and side supports visible (green color is before recent repainting); (b) side (red line) and central (thick, black line) support diagonals, with indication of horizontal reaction components; (c) bottom view around a central support; (d) detail picture of a node; (e) detail scheme around a node

Table 1: Bars' cross-section geometrical properties

\begin{tabular}{cccc}
\hline $\begin{array}{c}\text { Section } \\
\mathrm{n} .\end{array}$ & $\begin{array}{c}\text { Diameter / } \\
\text { thickness }(\mathrm{mm})\end{array}$ & $\begin{array}{c}\text { Area } \\
\left(\mathrm{cm}^{2}\right)\end{array}$ & $\begin{array}{c}\text { Radius of gyration } \\
(\mathrm{cm})\end{array}$ \\
\hline 1 & $76.1 / 3.2$ & 7.33 & 2.58 \\
2 & $88.9 / 3.6$ & 9.65 & 3.02 \\
3 & $101.6 / 3.6$ & 11.10 & 3.46 \\
4 & $114.3 / 3.6$ & 12.50 & 3.92 \\
5 & $133.0 / 4.0$ & 16.20 & 4.56 \\
6 & $159.0 / 4.5$ & 21.80 & 5.46 \\
7 & $159.0 / 7.1$ & 33.90 & 5.38 \\
8 & $193.7 / 10.0$ & 57.70 & 6.51 \\
\hline
\end{tabular}

different diameters and thicknesses listed in Table 1. It is of interest to put into evidence the planar location of each bar cross-section into the structure. Figure 3 shows the location of bar cross-section types in Table 1 on the whole structure,
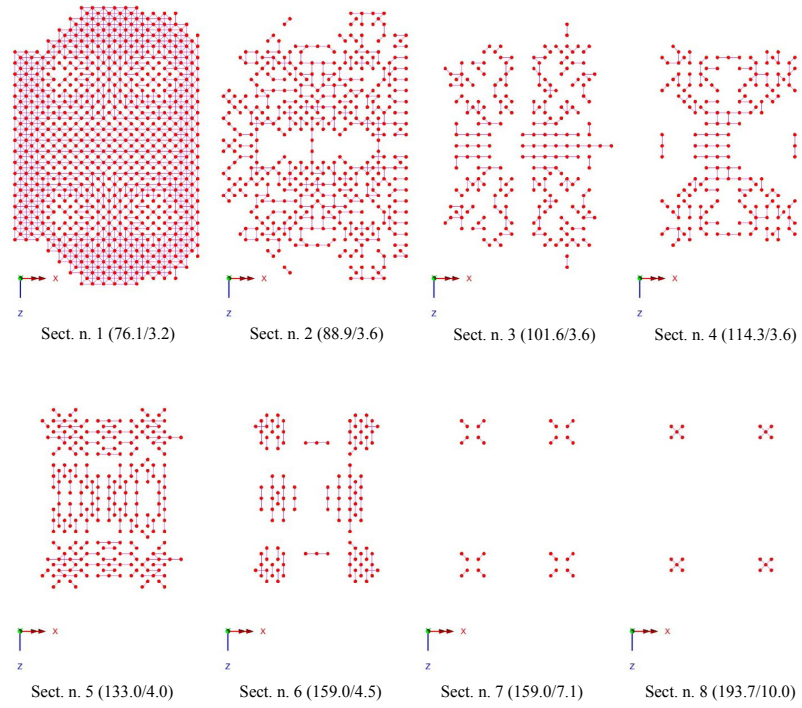

Figure 3: Planar location of bar cross-section types in Table 1 on the whole structure

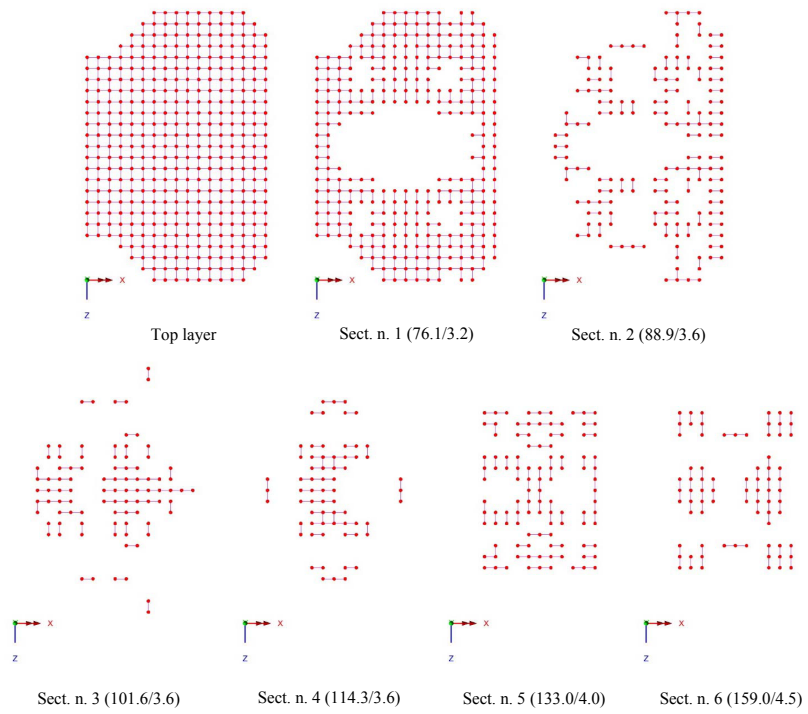

Figure 4: Planar location of bar cross-section types in Table 1 on the top layer

while Figures 4, 5, 6 and 7 show the placements on the top layer, bottom layer, diagonals and support diagonals, respectively.

Referring to the structural design report, the load combinations (LC) listed in Table 2 were considered.

The maximum nodal force, applied to the top layer nodes, was equal to $29.808 \mathrm{kN}$ for the inner nodes, and to its half for the edge nodes. Therefore, five main loading conditions were analyzed.

For each main loading condition, the most stretched bar and the most compressed bar among the top layer, the bottom layer and the diagonals, and the most compressed 
Table 2: Load combinations (LC) considered in the present study

\begin{tabular}{lr}
\hline LC n. & Load combinations \\
\hline LC 1 & self-weight + permanent load + snow \\
LC 2 & self-weight + permanent load + snow + uniform thermal variation $\left(+20^{\circ} \mathrm{C}\right)$ on the whole structure \\
LC 3 & self-weight + permanent load + snow + uniform thermal variation $\left(-20^{\circ} \mathrm{C}\right)$ on the whole structure \\
LC 4 & self-weight + permanent load + snow + non-uniform thermal variation $\left(+5^{\circ} \mathrm{C}\right)$ on the top layer bars only \\
LC 5 & self-weight + permanent load + snow + non-uniform thermal variation $\left(-5^{\circ} \mathrm{C}\right)$ on the top layer bars only \\
\hline
\end{tabular}

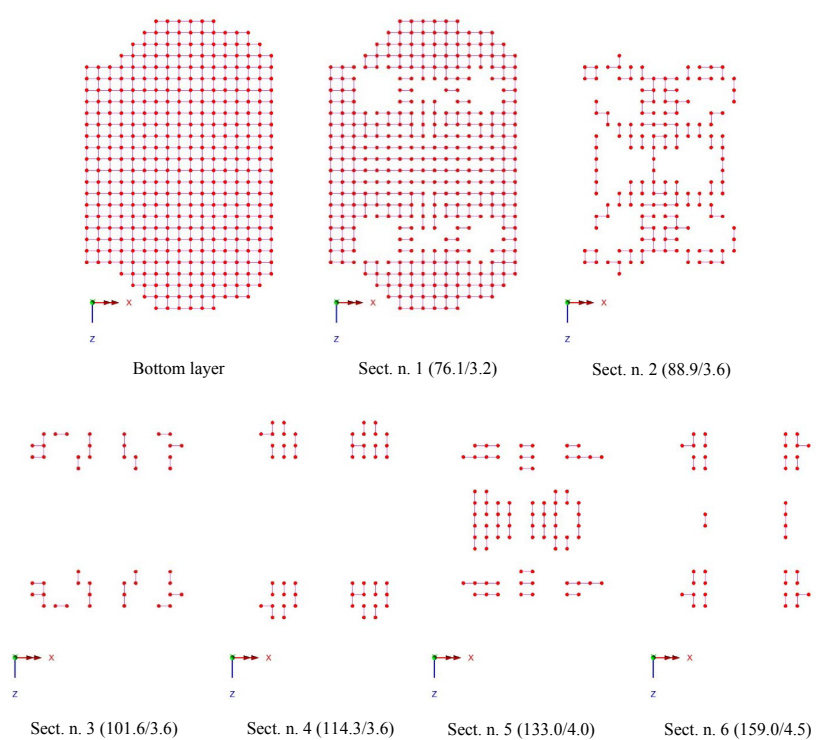

Figure 5: Planar location of bar cross-section types in Table 1 on the bottom layer

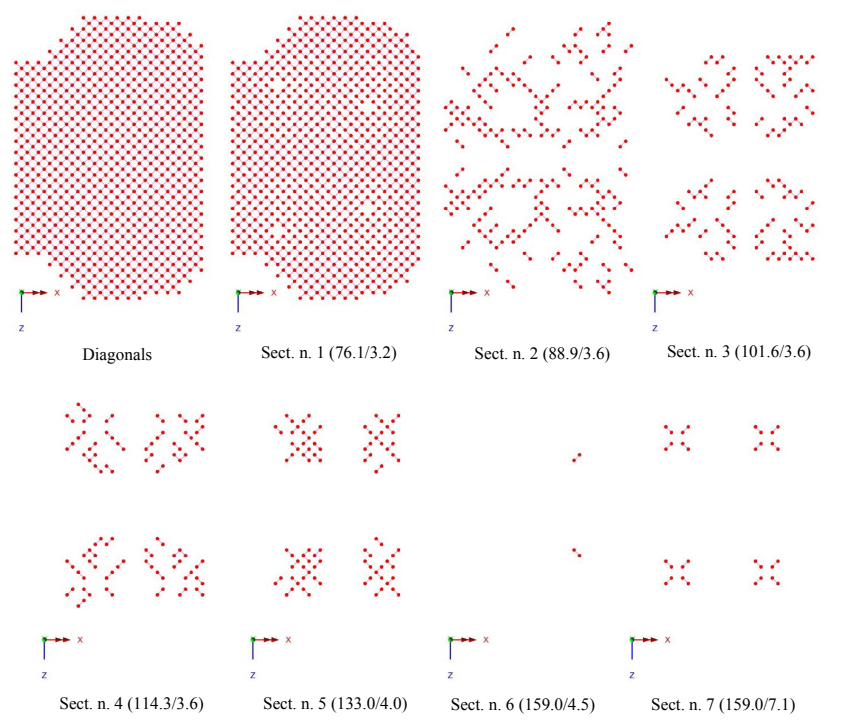

Figure 6: Planar location of bar cross-section types in Table 1 on the diagonals

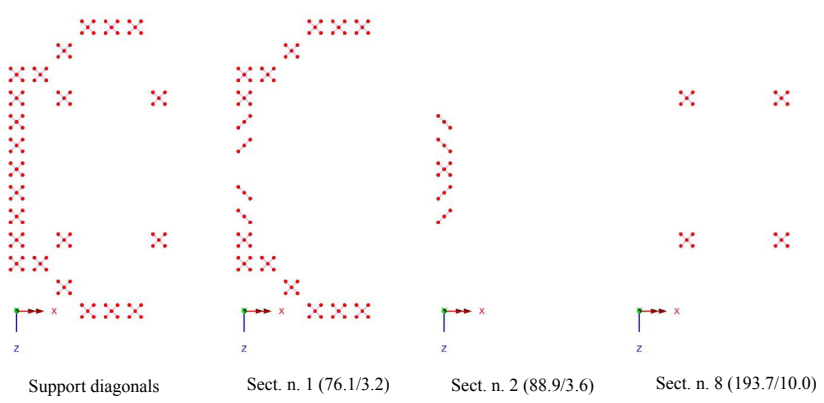

Figure 7: Planar location of bar cross-section types in Table 1 on the support diagonals

bar among the support diagonals, were alternately removed and the stress levels in the remaining bars checked. A total of 15 analyses with removal of the most stretched or the most compressed bar were run. In addition, the vertical displacements of five central nodes (one on the top and four on the bottom layer) were compared for the intact structure, the 15 situations with absence of the most stressed bars, and the situations with $50 \%$ reduction of axial stiffness of the same most stressed bars. The results of the analyses are presented in the next section.

\section{Results}

Figure 8 shows the most stressed bars in all the load combinations (see the black lines inside rectangle dashed boxes). In the following, even if not explicitly mentioned, we refer to axial stresses, only. The maximum tensile stress $\left(\sigma_{\max }^{+}\right)$ takes place among the bottom layer bars for LCs 1 and 2 (same most stretched bar, section type n. 2) and LCs 3 and 4 (same most stretched bar, section type n. 2), while it involves a top layer bar in LC 5 (section type n. 2). The maximum compressive stress $\left(\sigma_{\max }^{-}\right)$among top, bottom layers and diagonals, takes place in the same bottom layer bar for all LCs 1 to 5 (section type n. 6). Lastly, the maximum compressive stress among the support diagonals $\left(\sigma_{\max , \text { supp }}^{-}\right)$ takes place in the same central support bar for all LCs 1 to 5 (section type n. 8); see Figure 8. 
Table 3: Maximum / minimum stresses for all the load combinations (slanted numbers indicate percentage variation, $\Delta$, between values after and before (intact) bar removal; $\left.\Delta=\left(\sigma_{\text {rem }}-\sigma_{\text {intact }}\right) / \sigma_{\text {intact }} \times 100\right)$

\begin{tabular}{|c|c|c|c|c|c|c|c|}
\hline & & $\sigma_{\max }^{+}(\mathrm{MPa})$ & $\Delta(\%)$ & $\sigma_{\max }^{-}(\mathrm{MPa})$ & $\Delta(\%)$ & $\sigma_{\max , \text { supp }}^{-}(\mathrm{MPa})$ & $\Delta(\%)$ \\
\hline \multirow{4}{*}{ LC 1} & Intact & 227.587 & - & -171.232 & - & -191.685 & - \\
\hline & Rem max+ & 287.301 & 26.24 & -180.723 & 5.54 & -192.771 & 0.57 \\
\hline & Rem max- & 262.221 & 15.22 & -210.453 & 22.91 & -191.989 & 0.16 \\
\hline & Rem max-supp & 363.507 & 59.72 & -379.965 & 121.90 & -315.594 & 64.64 \\
\hline \multirow{4}{*}{ LC 2} & Intact & 227.282 & - & -171.878 & - & -192.101 & - \\
\hline & Rem max+ & 286.874 & 26.22 & -182.942 & 6.44 & -193.186 & 0.56 \\
\hline & Rem max- & 262.711 & 15.59 & -211.317 & 22.95 & -192.405 & 0.16 \\
\hline & Rem max-supp & 364.222 & 60.25 & -283.527 & 64.96 & -316.377 & 64.69 \\
\hline \multirow{4}{*}{ LC 3} & Intact & 228.325 & - & -170.586 & - & -191.270 & - \\
\hline & Rem max+ & 284.960 & 24.80 & -274.926 & 61.17 & -195.204 & 2.06 \\
\hline & Rem max- & 261.731 & 14.63 & -209.589 & 22.86 & -191.572 & 0.16 \\
\hline & Rem max-supp & 362.791 & 58.89 & -281.680 & 65.12 & -379.056 & 98.18 \\
\hline \multirow{4}{*}{ LC 4} & Intact & 233.971 & - & -165.749 & - & -189.292 & - \\
\hline & Rem max+ & 291.462 & 24.57 & -240.653 & 45.19 & -193.324 & 2.13 \\
\hline & Rem max- & 256.846 & 9.78 & -202.871 & 22.40 & -189.586 & 0.16 \\
\hline & Rem max-supp & 358.376 & 53.17 & -275.464 & 66.19 & -376.217 & 98.75 \\
\hline \multirow{4}{*}{ LC 5} & Intact & 222.937 & - & -176.715 & - & -194.078 & - \\
\hline & Rem max+ & 261.821 & 17.44 & -176.567 & -0.08 & -193.686 & -0.20 \\
\hline & Rem max- & 269.723 & 20.99 & -218.036 & 23.38 & -194.391 & 0.16 \\
\hline & Rem max-supp & 368.816 & 65.44 & -289.903 & 64.05 & -383.904 & 97.81 \\
\hline
\end{tabular}
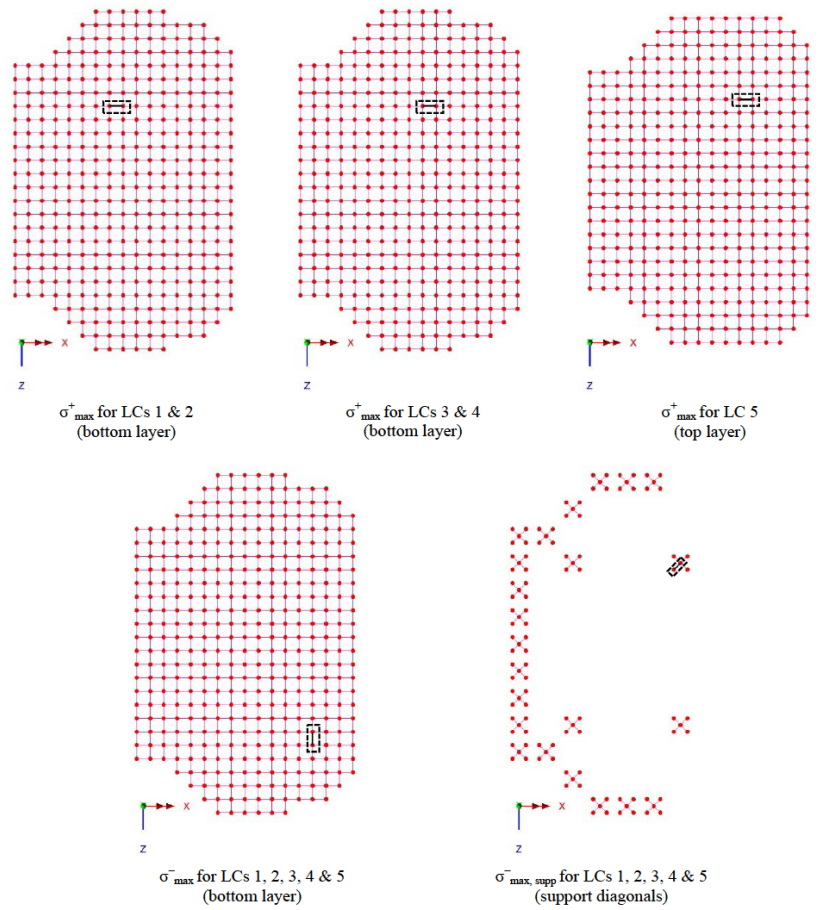

Figure 8: Planar location of the most stressed bars in all the load combinations
The bars indicated in Figure 8 are the elements that were alternately removed to investigate the stress increments. The results are in Table 3, where the maximum tensile and compressive stresses before and after the bar removal are compared for each load combination. The removals of the most stretched bar, the most compressed bar, and the most compressed bar among the support diagonals, are indicated as "Rem max+", "Rem max-", and "Rem max- supp", respectively. In the same table, the percentage difference, $\Delta$, between the values after and before the bar removal are reported with slanted character.

The values in Table 3 indicate that higher stress increments are produced by support diagonal removal, always followed by removal of the most stretched bar, with one exception only in favor of the most compressed bar (see LC 5 in Table 3). Percentage stress increments obtained removing the most compressed support diagonal, are all above $50 \%$, with one case overcoming $120 \%$. The maximum stress increment obtained by removing the most stretched / compressed bar among the other elements is sligtly above $60 \%$, followed by one case around $45 \%$ and all the other increments remaining below $30 \%$, although not negligible in general. As an example, in Figures 9 to 12 the stress contour plots before and after bar removal are compared for LC 1 . The most stressed elements are comprised between 

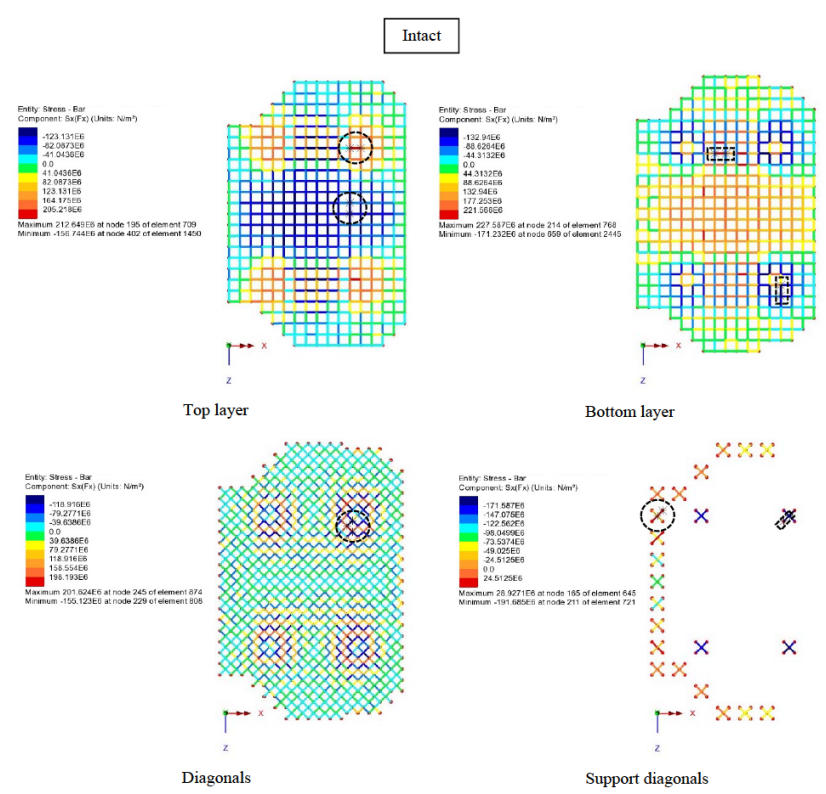

Figure 9: Stress contour plots before bar removal for LC 1 (red = tension; blue = compression)

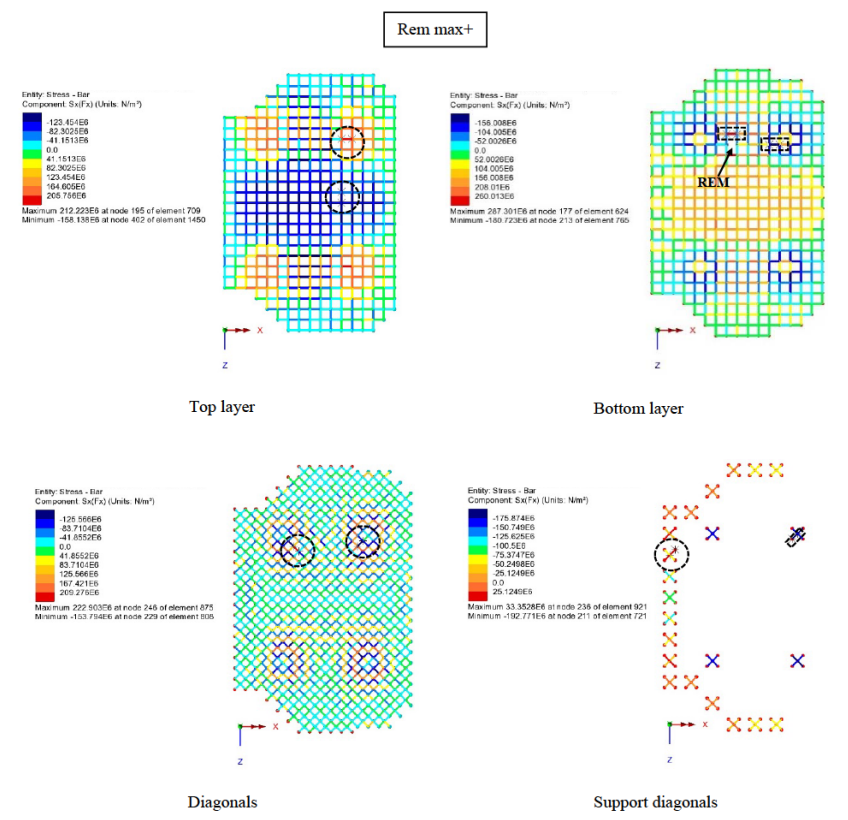

Figure 10: Stress contour plots after most tense bar removal for LC 1 (red $=$ tension; blue $=$ compression $)$

$\times$ symbols and included inside dashed circles, in general, or inside dashed rectangles if they are absolutely the most stressed ones among the top and bottom layers and the support diagonals. In particular: Figure 9 shows the stress contur plots for the intact structure (Intact); Figure 10 refers to the removal of the most stretched bar (Rem max+); Figure 11 refers to the removal of the most compressed bar (Rem max-); Figure 12 is related to the removal of the most

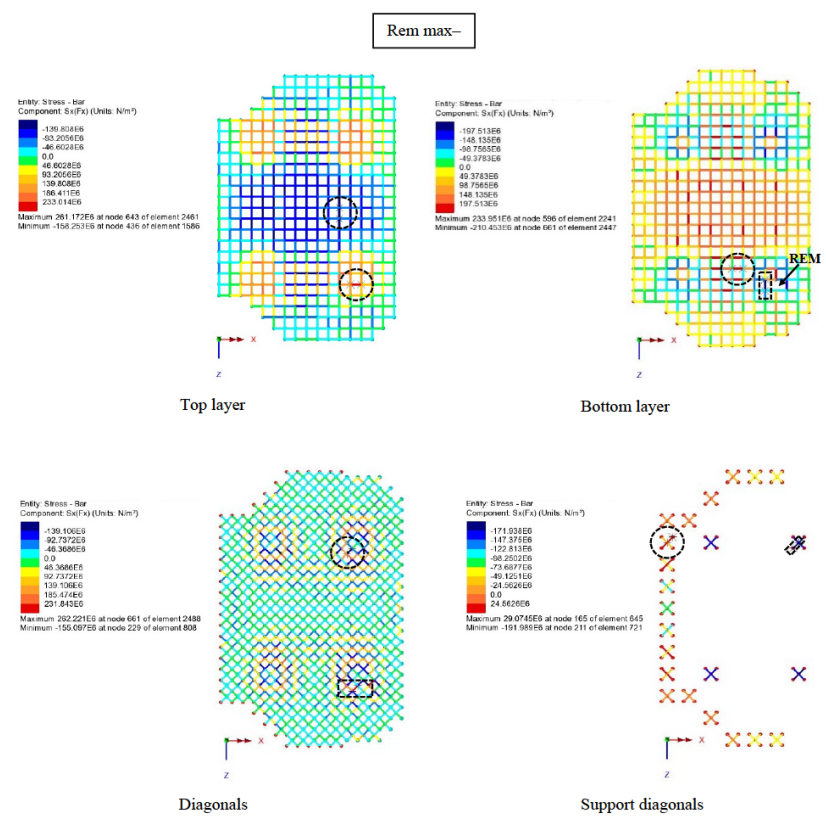

Figure 11: Stress contour plots after most compressed bar removal for LC 1 (red = tension; blue = compression)

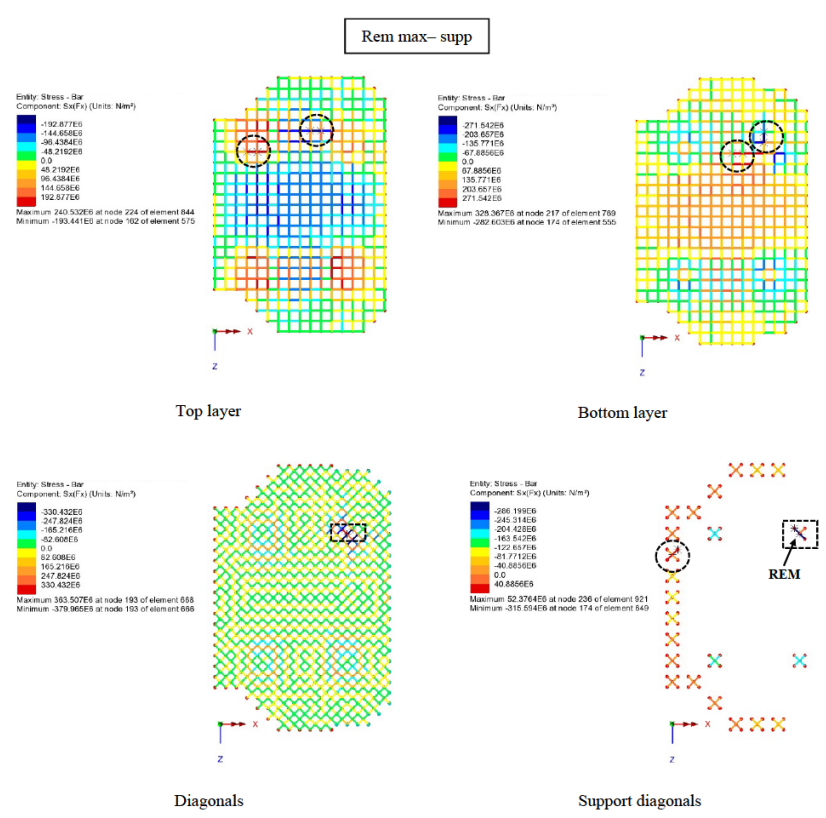

Figure 12: Stress contour plots after most compressed support bar removal for $\mathrm{LC} 1$ (red = tension; blue $=$ compression)

compressed support diagonal (Rem max- supp). As can be seen from Figures 9-12, there is no remarkable stress redistribution; conversely, stresses concentrate in the weaker regions, with the highest stresses taking place, in general, in bars which are close to the removed ones. Similar stress contour plots, not shown here for the sake of brevity, are obtained for the other load conditions. 
Table 4: Vertical displacements, DY, of control points PT, PB1-PB4 (positive value means downward) for different load combinations and damage levels (slanted numbers indicate percentage variation, $\Delta$, between values after and before (intact) bar removal / damage; $\left.\Delta=\left(D Y_{\text {rem } / 0.5 A}-D Y_{\text {intact }}\right) / D Y_{\text {intact }} \times 100\right)$

\begin{tabular}{|c|c|c|c|c|c|c|c|c|c|c|c|}
\hline & & \multicolumn{2}{|c|}{ PT } & \multicolumn{2}{|c|}{ PB1 } & \multicolumn{2}{|c|}{ PB2 } & \multicolumn{2}{|c|}{ PB3 } & \multicolumn{2}{|l|}{ PB4 } \\
\hline & & $D Y(\mathrm{~m})$ & $\Delta(\%)$ & $D Y(\mathrm{~m})$ & $\Delta(\%)$ & $D Y(\mathrm{~m})$ & $\Delta(\%)$ & $D Y(\mathrm{~m})$ & $\Delta(\%)$ & $D Y(\mathrm{~m})$ & $\Delta(\%)$ \\
\hline & Intact & 0.145461 & - & 0.144525 & & 0.142789 & - & 0.142735 & - & 0.144422 & - \\
\hline & Rem max+ & 0.146392 & 0.64 & 0.145610 & 0.75 & 0.143803 & 0.71 & 0.143512 & 0.54 & 0.145263 & 0.58 \\
\hline & Rem max- & 0.147675 & 1.52 & 0.146454 & 1.33 & 0.144962 & 1.52 & 0.145234 & 1.75 & 0.146612 & 1.52 \\
\hline \multirow[t]{7}{*}{ LC 1} & Rem max- supp & 0.162838 & 11.95 & 0.162320 & 12.31 & 0.162517 & 13.82 & 0.159009 & 11.40 & 0.159545 & 10.47 \\
\hline & $0.5 A \max +$ & 0.145646 & 0.13 & 0.144741 & 0.15 & 0.142991 & 0.14 & 0.142890 & 0.11 & 0.144589 & 0.12 \\
\hline & $0.5 A \mathrm{max}-$ & 0.145777 & 0.22 & 0.144801 & 0.19 & 0.143100 & 0.22 & 0.143093 & 0.25 & 0.144735 & 0.22 \\
\hline & $0.5 A$ max- supp & 0.146148 & 0.47 & 0.145228 & 0.49 & 0.143569 & 0.55 & 0.143379 & 0.45 & 0.145020 & 0.41 \\
\hline & Intact & 0.144035 & - & 0.143868 & - & 0.14214 & - & 0.142085 & - & 0.143765 & - \\
\hline & Rem max+ & 0.144965 & 0.65 & 0.144952 & 0.75 & 0.143152 & 0.71 & 0.142861 & 0.55 & 0.144605 & 0.58 \\
\hline & Rem max- & 0.146258 & 1.54 & 0.145804 & 1.35 & 0.144321 & 1.53 & 0.144593 & 1.77 & 0.145963 & 1.53 \\
\hline \multirow[t]{7}{*}{ LC 2} & Rem max- supp & 0.161445 & 12.09 & 0.161695 & 12.39 & 0.161904 & 13.90 & 0.161904 & 13.95 & 0.158389 & 10.17 \\
\hline & $0.5 A \max +$ & 0.144179 & 0.10 & 0.144036 & 0.12 & 0.142296 & 0.11 & 0.142205 & 0.08 & 0.143895 & 0.09 \\
\hline & $0.5 A \max -$ & 0.144446 & 0.29 & 0.144226 & 0.25 & 0.142543 & 0.28 & 0.142550 & 0.33 & 0.144172 & 0.28 \\
\hline & $0.5 A$ max- supp & 0.144904 & 0.60 & 0.144757 & 0.62 & 0.143126 & 0.69 & 0.142900 & 0.57 & 0.144521 & 0.53 \\
\hline & Intact & 0.146886 & - & 0.145182 & - & 0.143439 & - & 0.143385 & - & 0.145079 & - \\
\hline & Rem max+ & 0.147768 & 0.60 & 0.146188 & 0.69 & 0.144439 & 0.70 & 0.144127 & 0.52 & 0.145849 & 0.53 \\
\hline & Rem max- & 0.149092 & 1.50 & 0.147103 & 1.32 & 0.145604 & 1.51 & 0.145874 & 1.74 & 0.147261 & 1.50 \\
\hline \multirow[t]{7}{*}{ LC 3} & Rem max- supp & 0.164231 & 11.81 & 0.162944 & 12.23 & 0.163130 & 13.73 & 0.159628 & 11.33 & 0.160174 & 10.40 \\
\hline & $0.5 A \max +$ & 0.147101 & 0.15 & 0.154428 & 6.37 & 0.143684 & 0.17 & 0.143566 & 0.13 & 0.145267 & 0.13 \\
\hline & $0.5 A$ max- & 0.147108 & 0.15 & 0.145376 & 0.13 & 0.143658 & 0.15 & 0.143636 & 0.18 & 0.145299 & 0.15 \\
\hline & $0.5 A$ max- supp & 0.147391 & 0.34 & 0.145699 & 0.36 & 0.144012 & 0.40 & 0.143858 & 0.33 & 0.145518 & 0.30 \\
\hline & Intact & 0.145571 & - & 0.14471 & - & 0.143324 & - & 0.142982 & - & 0.144252 & - \\
\hline & Rem max+ & 0.144969 & -0.41 & 0.144034 & -0.47 & 0.142434 & -0.62 & 0.142120 & -0.60 & 0.143699 & -0.38 \\
\hline & Rem max- & 0.146209 & 0.44 & 0.144870 & 0.11 & 0.143512 & 0.13 & 0.143778 & 0.56 & 0.145029 & 0.54 \\
\hline \multirow[t]{7}{*}{ LC 4} & Rem max-supp & 0.161154 & 10.70 & 0.160493 & 10.91 & 0.160809 & 12.20 & 0.157368 & 10.06 & 0.157781 & 9.38 \\
\hline & $0.5 A \max +$ & 0.144247 & -0.91 & 0.143209 & -1.04 & 0.141614 & -1.19 & 0.141512 & -1.03 & 0.143067 & -0.82 \\
\hline & $0.5 A \mathrm{max}-$ & 0.144373 & -0.82 & 0.143270 & -1.00 & 0.141710 & -1.13 & 0.141706 & -0.89 & 0.143213 & -0.72 \\
\hline & $0.5 A \max -\mathrm{supp}$ & 0.144744 & -0.57 & 0.143697 & -0.70 & 0.142179 & -0.80 & 0.141995 & -0.69 & 0.143500 & -0.52 \\
\hline & Intact & 0.146855 & - & 0.146047 & - & 0.144170 & - & 0.144111 & - & 0.145935 & - \\
\hline & Rem max+ & 0.147418 & 0.38 & 0.146660 & 0.42 & 0.144796 & 0.48 & 0.144617 & 0.35 & 0.146436 & 0.34 \\
\hline & Rem max- & 0.149140 & 1.56 & 0.148037 & 1.36 & 0.146412 & 1.60 & 0.146689 & 1.79 & 0.148195 & 1.55 \\
\hline \multirow[t]{4}{*}{ LC 5} & Rem max- supp & 0.164648 & 12.12 & 0.164285 & 12.49 & 0.164349 & 14.04 & 0.160761 & 11.55 & 0.161434 & 10.62 \\
\hline & $0.5 A \max +$ & 0.146987 & 0.09 & 0.146191 & 0.10 & 0.144317 & 0.14 & 0.144230 & 0.08 & 0.146052 & 0.08 \\
\hline & $0.5 A \max -$ & 0.147182 & 0.22 & 0.146332 & 0.20 & 0.144491 & 0.26 & 0.144480 & 0.26 & 0.146258 & 0.22 \\
\hline & $0.5 A$ max - supp & 0.147551 & 0.47 & 0.146759 & 0.49 & 0.144960 & 0.59 & 0.144762 & 0.45 & 0.146540 & 0.41 \\
\hline
\end{tabular}

To investigate the effect of bar stiffness reduction or bar removal on the global structural flexibility, five displacement control points were selected: one on the top layer, PT, and four on the bottom layer, PB1, PB2, PB3 and PB4; see Figure 13. These represent the points of maximum vertical displacement. In Table 4, the vertical displacement of the control points are shown for the intact structure, the $15 \mathrm{situ}-$ ations with absence of the most stressed bars, and the situations with $50 \%$ reduction of cross-section area of the same most stressed bars (indicated as "0.5A max+", "0.5A max-", and "0.5A max-supp"). Displacements are expressed in $\mathrm{m}$, positive value means downward, while slanted numbers indicate the percentage difference between the values after and before the bar removal / cross-section reduction.
From the comparisons in Table 4, we see that higher displacement increases correspond to diagonal support bar removal, followed by removal of compression bars and, then, of tension bars. Percentage variations are comprised between 9 and $15 \%$ for the removal of support bars, are of order $1 \%$ for the removal of other compression bars (exception made for LC 4), and of order $0.1 \%$ for the removal of tension bars. Percentage increments of displacement are all below $1 \%$ for the situations with $50 \%$ reduction of cross-section area, with prevailing effect for support diagonal bars. Lastly, by comparing the displacement increments corresponding to $50 \%$ reduction of bar stiffness and to complete bar removal, we recognize a nonlinear, softening response of the structure stiffness with damage severity. 
Table 5: Geometric and buckling properties of bars

\begin{tabular}{|c|c|c|c|c|c|c|c|}
\hline $\begin{array}{c}\text { Section } \\
\mathrm{n} .\end{array}$ & $\begin{array}{l}\text { Diameter / } \\
\text { thickness } \\
(\mathrm{mm})\end{array}$ & $\begin{array}{c}\text { Area, } \\
A \\
\left(\mathrm{~cm}^{2}\right)\end{array}$ & $\begin{array}{l}\text { Radius of } \\
\text { gyration, } \rho \\
\text { (cm) }\end{array}$ & $\begin{array}{l}\text { Element } \\
\text { length, } l \\
(\mathrm{~cm})\end{array}$ & $\begin{array}{c}\text { Slenderness } \\
\text { ratio, } l / \rho \\
(-)\end{array}$ & $\begin{array}{l}\text { Euler's buckling } \\
\text { load, } P_{c} \\
(\mathrm{kN})\end{array}$ & $\begin{array}{l}\text { Euler's buckling } \\
\text { pressure, } \sigma_{c} \\
(\mathrm{MPa})\end{array}$ \\
\hline \multirow{2}{*}{1} & \multirow{2}{*}{$76.1 / 3.2$} & \multirow{2}{*}{7.33} & \multirow{2}{*}{2.58} & 360 & 139.5 & 78.0 & 106.5 \\
\hline & & & & 411 & 159.3 & 59.9 & 81.7 \\
\hline \multirow{2}{*}{2} & \multirow{2}{*}{88.9 / 3.6} & \multirow{2}{*}{9.65} & \multirow{2}{*}{3.02} & 360 & 119.2 & 140.8 & 145.9 \\
\hline & & & & 411 & 136.1 & 108.0 & 111.9 \\
\hline \multirow{2}{*}{3} & \multirow{2}{*}{101.6 / 3.6} & \multirow{2}{*}{11.1} & \multirow{2}{*}{3.46} & 360 & 104.0 & 212.5 & 191.5 \\
\hline & & & & 405 & 117.1 & 167.9 & 151.3 \\
\hline \multirow{2}{*}{4} & \multirow{2}{*}{$114.3 / 3.6$} & \multirow{2}{*}{12.5} & \multirow{2}{*}{3.92} & 360 & 91.8 & 307.2 & 245.7 \\
\hline & & & & 411 & 104.8 & 235.7 & 188.5 \\
\hline \multirow{2}{*}{5} & \multirow{2}{*}{$133.0 / 4.0$} & \multirow{2}{*}{16.2} & \multirow{2}{*}{4.56} & 360 & 78.9 & 538.7 & 332.5 \\
\hline & & & & 411 & 90.1 & 413.3 & 255.1 \\
\hline \multirow{2}{*}{6} & \multirow{2}{*}{$159.0 / 4.5$} & \multirow{2}{*}{21.8} & \multirow{2}{*}{5.46} & 360 & 65.9 & 1039.3 & 476.8 \\
\hline & & & & 411 & 75.3 & 797.4 & 365.8 \\
\hline 7 & $159.0 / 7.1$ & 33.9 & 5.38 & 411 & 76.4 & 1203.9 & 355.1 \\
\hline 8 & $193.7 / 10.0$ & 57.7 & 6.51 & 302.5 & 46.5 & 5538.7 & 959.9 \\
\hline
\end{tabular}
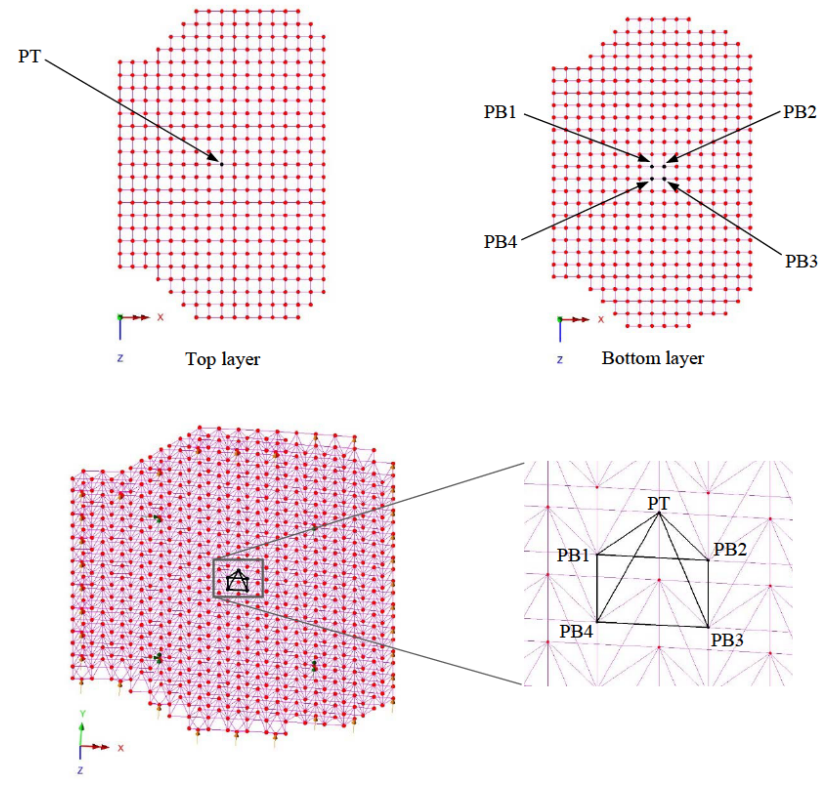

Figure 13: Control points for comparison of vertical displacements in the different load combinations and damage levels

To further investigate the effect of local bar removals on a global loss of stiffness, linearized buckling analyses were run. In Table 5 are reported, for each bar section type, the cross-sectional area $A$, the radius of gyration $\rho$, the typical (maximum / minimum) element length $l$, the slenderness ratio $l / \rho$, the Euler's buckling load $P_{c}=\pi^{2} E A /(l / \rho)^{2}$, and the Euler's buckling pressure $\sigma_{c}=P_{c} / A$. Being the bars hinged at their ends, the equivalent buckling length coincides with the bar length $l$; see [14].
Table 6 collects the results of all the linearized buckling analyses conducted, with indicated the critical load multiplier $\lambda_{c}$ and the most critical element. The analyses were performed by considering the bars deformable both in axial direction and in bending (each bar was modelled through six Euler-Bernoulli beam elements). As can be seen, the safety factor with respect to Euler buckling is about 1.3 for the intact structure and about 0.2 when the support diagonal is removed, in all the LCs. For the other element removals, the safety factor is comprised between about 0.4 and 0.8 (see Table 6). In the analyzed case, the first buckling load is attained when the compressive axial load reaches the buckling load in the most critical element. This mechanism activates much before a global buckling mode, involving the structure as a whole or large portions of it, can be activated. The critical elements are always bars with cross-section type n. 1 (76.1 / $3.2 \mathrm{~mm}$ ), with length equal to $411 \mathrm{~cm}$ in cases when a support diagonal is removed, or equal to $360 \mathrm{~cm}$ in all the other cases (see Table 6). In principle, a permanent monitoring system, as well as occasional measurements, could be set up on the most stressed elements in order to prevent buckling instability. For example, among other methods, resonant frequency measurements can be used to make experimental predictions on the buckling load of compressed elements [15-17].

To investigate the order of magnitude of the critical load multiplier for a global buckling mode, where local buckling in single elements is not involved, linearized buckling analyses were run on a truss model made up by bar finite elements (axial behavior only, bending behavior inhibited). 
Table 6: Critical load multipliers $\lambda_{c}$ for linearized buckling analysis

\begin{tabular}{|c|c|c|c|}
\hline & & Critical load multiplier, $\lambda_{c}(-)$ & Critical element \\
\hline \multirow{4}{*}{ LC 1} & Intact & 1.30 & Sect. n. $1, l=360 \mathrm{~cm}$ \\
\hline & Rem max+ & 0.59 & Sect. n. $1, l=360 \mathrm{~cm}$ \\
\hline & Rem max- & 0.74 & Sect. n. $1, l=360 \mathrm{~cm}$ \\
\hline & Rem max-supp & 0.21 & Sect. n. $1, l=411 \mathrm{~cm}$ \\
\hline \multirow{4}{*}{ LC 2} & Intact & 1.31 & Sect. n. $1, l=360 \mathrm{~cm}$ \\
\hline & Rem max+ & 0.58 & Sect. n. $1, l=360 \mathrm{~cm}$ \\
\hline & Rem max- & 0.73 & Sect. n. $1, l=360 \mathrm{~cm}$ \\
\hline & Rem max- supp & 0.21 & Sect. n. $1, l=411 \mathrm{~cm}$ \\
\hline \multirow{4}{*}{ LC 3} & Intact & 1.29 & Sect. n. $1, l=360 \mathrm{~cm}$ \\
\hline & Rem max+ & 0.43 & Sect. n. $1, l=360 \mathrm{~cm}$ \\
\hline & Rem max- & 0.75 & Sect. n. $1, l=360 \mathrm{~cm}$ \\
\hline & Rem max-supp & 0.22 & Sect. n. $1, l=411 \mathrm{~cm}$ \\
\hline \multirow{4}{*}{ LC 4} & Intact & 1.29 & Sect. n. $1, l=360 \mathrm{~cm}$ \\
\hline & Rem max+ & 0.44 & Sect. n. $1, l=360 \mathrm{~cm}$ \\
\hline & Rem max- & 0.81 & Sect. n. $1, l=360 \mathrm{~cm}$ \\
\hline & Rem max- supp & 0.22 & Sect. n. $1, l=411 \mathrm{~cm}$ \\
\hline \multirow{4}{*}{ LC 5} & Intact & 1.31 & Sect. n. $1, l=360 \mathrm{~cm}$ \\
\hline & Rem max+ & 0.69 & Sect. n. $1, l=360 \mathrm{~cm}$ \\
\hline & Rem max- & 0.68 & Sect. n. $1, l=360 \mathrm{~cm}$ \\
\hline & Rem max-supp & 0.21 & Sect. n. $1, l=411 \mathrm{~cm}$ \\
\hline
\end{tabular}

In this case, the critical load multiplier is of order 20 when a support diagonal bar is removed, and of order $\sim 90$ in all the other cases, regardless of the LC. Therefore, this kind of global buckling modes are only virtual, and local (element) buckling of compressed bars is crucial. On the other hand, in general, possible interactions between local and global buckling modes can be investigated by large-displacement nonlinear analyses $[18,19]$. However, these kind of phenomena are more preeminent for single layer grid shells than for double layer truss roofs like the one analyzed here. For the former, in fact, geometric stiffness due to initial curvature is relevant, and geometric nonlinearities are in general non-negligible even for low loading levels. In such cases, snap-through buckling and interaction phenomena between snap-trough and Euler instability may become crucial [19]. Conversely, global geometric stiffness is relatively of little importance in double layer space trusses.

Referring to the stress values in Table 3 for the intact structure, the minimum safety factors with respect to tensile yielding and failure are respectively equal to 1.52 and 2.18. When a bar is removed, they become respectively $0.96(-36.84 \%)$ and $1.38(-36.70 \%)$ in the worst case, 1.38 $(-9.21 \%)$ and $1.99(-8.72 \%)$ in the best case. Notice that, while the yield stress is overcome in some cases, the value of tensile strength is never reached in the analyzed situations. Values in Tables 3 and 6 indicate that the situation of total absence of the most stretched or most compressed bar is to be judged as not admissible, since it could impair bearing capacity in at least one other element. On the other hand, this also points out that understanding if structure is able or not to withstand the previous extreme condition without collapsing, requires a nonlinear collapse analysis. The latter, however, is beyond the aim of the present study.

In the previous investigation, slow bar removals were considered, i.e., without including dynamic amplification effects due to possible sudden local failures. To identify the order of magnitude of such possible dynamic amplification effects, some additional analyses were conducted. Table 7 shows the results obtained when the nodal force was doubled $(29.808 \times 2=59.616 \mathrm{kN})$ for the six top nodes closest to the removed bar (those above the two end nodes of the removed element); compare the stress increments in Tables 3 and 7.

Lastly, given the sort of inner periodicity of the construction module, a preliminary investigation was also conducted to understand if there is a typical distance after which the removal effect disappears. Actually, it must be observed that, even if the dimensions of the moduli are the same, the cross-sections of the bars composing the different moduli change. However, it was found that, in general, stress variations less than $5 \%$ are detected after a distance equivalent to five moduli, although with some exceptions. 
Table 7: Maximum / minimum stresses when a dynamic amplification factor is considered (slanted numbers indicate percentage variation, $\Delta$, between values after and before (intact) bar removal; $\left.\Delta=\left(\sigma_{\text {rem }}-\sigma_{\text {intact }}\right) / \sigma_{\text {intact }} \times 100\right)$

\begin{tabular}{llcccccc}
\hline & & $\sigma_{\max }^{+}(\mathrm{MPa})$ & $\Delta(\%)$ & $\sigma_{\max }^{-}(\mathrm{MPa})$ & $\Delta(\%)$ & $\sigma_{\text {max }, \text { supp }}^{-}(\mathrm{MPa})$ & $\Delta(\%)$ \\
\hline \multirow{4}{*}{ LC 1 } & Intact & 227.587 & - & -171.232 & - & -191.685 & - \\
& Rem max+ & 338.194 & 48.60 & -206.083 & 20.35 & -200.808 & 4.76 \\
& Rem max- & 263.021 & 15.57 & -208.921 & 22.01 & -194.913 & 1.68 \\
& Rem max- supp & 371.847 & 63.39 & -426.127 & 148.86 & -340.936 & 77.86 \\
& Intact & 227.282 & - & -171.878 & - & -192.101 & - \\
LC 2 & Rem max+ & 337.668 & 48.57 & -208.261 & 21.17 & -201.210 & 4.74 \\
& Rem max- & 263.512 & 15.94 & -209.785 & 22.05 & -195.441 & 1.74 \\
& Rem max- supp & 372.589 & 63.93 & -427.037 & 148.45 & -341.722 & 77.89 \\
& Intact & 228.325 & - & -170.586 & - & -191.27 & - \\
LC 3 & Rem max+ & 331.601 & 45.23 & -283.802 & 66.37 & -209.016 & 9.28 \\
& Rem max- & 255.372 & 11.85 & -206.344 & 20.96 & -195.444 & 2.18 \\
& Rem max- supp & 371.159 & 62.56 & -425.218 & 149.27 & -340.155 & 77.84 \\
& Intact & 233.971 & - & -165.749 & - & -189.292 & - \\
LC 4 & Rem max+ & 338.104 & 44.51 & -276.529 & 66.84 & -207.136 & 9.43 \\
& Rem max- & 255.826 & 9.34 & -199.625 & 20.44 & -192.776 & 1.84 \\
& Rem max- supp & 373.118 & 59.47 & -422.236 & 154.74 & -336.117 & 77.57 \\
& Intact & 222.937 & - & -176.715 & - & -194.078 & - \\
LC 5 & Rem max+ & 264.695 & 18.73 & -179.928 & 1.82 & -205.893 & 6.09 \\
& Rem max- & 265.207 & 18.96 & -214.790 & 21.55 & -199.167 & 2.62 \\
& Rem max- supp & 377.211 & 69.20 & -430.102 & 143.39 & -345.753 & 78.15 \\
\hline
\end{tabular}

Therefore, a general conclusion cannot be drawn at this stage and the matter deserves further deepening in future studies.

\section{Discussion and conclusions}

A robustness analysis of an existing airport space frame structure was conducted by a linear finite element model to investigate the global response to the failure (removal) of a key element. As it was done in the original structural design, the structure was modeled as an ideal space truss composed of linearly elastic, perfectly hinged bars. With respect to five basic loading conditions (selected as the worst among those considered in the original structural report), fifteen situations with alternative removal of the most stretched or the most compressed bar were considered. Based on the results obtained, the following special conclusions can be drawn for the analyzed problem:

1. In none of the analyzed cases the removal of the most stressed bar is sufficient to transform the whole structure, or part of it, into a mechanism.

2. There is no remarkable stress redistribution; conversely, stresses concentrate in the weaker regions, with the highest stresses taking place, in general, in bars which are close to the removed ones.

3. Among the loading conditions examined, there is always at least one case in which the removal of the most stretched or compressed element implies the overcoming of the tensile yield stress ( $355 \mathrm{MPa}$ ) and/or of the buckling load in the most stressed remaining bars.

4. Based on the previous Point 3, the situation of total absence of the most stretched or compressed bar is judged as not admissible, since it could impair bearing capacity in at least one other element.

5. Understanding whether or not the structure is able to withstand the previous extreme condition without collapsing would require a step-by-step nonlinear analysis.

In addition to the previous specific considerations, some more general conclusions can be gathered from the present study.

Concerning the effect of bar stiffness reduction or bar removal on the global structural flexibility, we found a nonlinear, softening response of the structure with increasing of damage level. In other words, displacements obtained by halving the axial stiffness of a bar, result more than doubled if the same bar is totally removed. In general, much 
higher displacement increases are found when damage, or removal, regard support diagonal bars; compression bars follow, while tension bars have the smallest effect.

Also, we remark that percentage increases in stresses are one order of magnitude larger than percentage increases in displacements: the latter are of order $10^{1}$ for removal of support diagonals and of order $10^{0}-10^{-1}$ in the other cases. This fact must be well taken into account in a possible structural monitoring, i.e., detecting anomalous variations in bar strains would be easier than detecting anomalous variations in nodal displacements.

In general, the present investigation points out that space trusses like the one considered here, often used for large airport station roofs because they combine high structural efficiency and lightweight, are very sensitive to local losses, since their redistribution capabilities are not large. On the other hand, the double layer scheme ensures a relatively high global stiffness, thus global buckling is governed by local (bar) buckling, attained when the compressive axial load reaches the buckling load in the most critical element. This mechanism activates much before a global buckling mode, involving the structure as a whole or large portions of it, can be activated.

To conduct a more accurate nonlinear collapse analysis, both material and geometric nonlinearities should be considered. In this case, appropriate modelling of connection nodes should also be included, since it has a strong influence on the global response, and therefore, on the final collapse mechanism and load. However, this represents a crucial point, because of imperfection sensitivity: when analyzing a complex existing structure, the possible discrepancies between the numerical model and the real situation may produce non-negligible differences between the model predictions and the actual structural behavior. Therefore, sensitivity and parametric analyses should be carried out. All these aspects go far beyond the scope of the present study.

Funding information: The authors state no funding involved.

Author contributions: All authors have accepted responsibility for the entire content of this manuscript and approved its submission.

Conflict of interest: The authors state no conflict of interest.

\section{References}

[1] Starossek U, Haberland M. Approaches to measures of structural robustness. Struct Infrastruct Eng. 2012;7(7-8):625-31.

[2] Baker JW, Schubert M, Faber MH. On the assessment of robustness. Struct Saf. 2008;30(3):253-67.

[3] Comité Européen de Normalisation, Eurocode 0 - Basics of structural design; 2002.

[4] De Biagi V, Chiaia B. Robustness and complexity of frame structures. Int J Solids Struct. 2013;50:3723-41.

[5] Adam JM, Parisi F, Sagaseta J, Lu X. Research and practice on progressive collapse and robustness of building structures in the 21st century. Eng Struct. 2018;173:122-49.

[6] Consiglio Nazionale delle Ricerche, CNR-DT 214/2018 Istruzioni per la valutazione della robustezza sulle costruzioni; 2018 (in Italian).

[7] Hanaor A. Design and Behavior of Reticulated Spatial Structural Systems. Int J Space Structures. 1995;10(3):139-49.

[8] Pieraccini L, Palermo M, Trombetti T, Baroni F. The role of ductility in the collapse of a long-span steel roof in North Italy. Eng Fail Anal. 2017;82:243-65.

[9] Karoki Kabando E, Gong J. An overview of long-span spatial grid structures failure case studies. Asian J. Civil Eng. 2019;20(8):1137-52.

[10] Vatansever C. Investigation of buckled truss bars of a space truss roof system. Eng Fail Anal. 2019;106:104156.

[11] Tian LM, Wei JP, Hao JP. Optimisation of long-span single-layer spatial grid structures to resist progressive collapse. Eng Struct. 2019;188:394-405.

[12] Wei JP, Tian LM, Hao JP. Improving the progressive collapse resistance of long-span single-layer spatial grid structures. Constr Build Mater. 2018;171:96-108.

[13] Tian LM, Nie XN, Zhong WH, Wei JP. Comparison of the progressive collapse resistances of different single-layer latticed domes. J Construct Steel Res. 2019;162:105697.

[14] Bazant ZP, Cedolin L. Stability of Structures. New York: Oxford University Press; 1991.

[15] Carpinteri A, Malvano R, Manuello A, Piana G. Fundamental frequency evolution in slender beams subjected to imposed axial displacements. J Sound Vibrat. 2014;333(11):2390-403.

[16] Piana G, Lofrano E, Manuello A, Ruta G. Natural frequencies and buckling of compressed non-symmetric thin-walled beams. Thinwalled Struct. 2017;111:189-96.

[17] Piana G, Lofrano E, Manuello A, Ruta G, Carpinteri A. Compressive buckling for symmetric TWB with non-zero warping stiffness. Eng Struct. 2017;135:246-58.

[18] Carpinteri A, Bazzucchi F, Manuello A. Nonlinear instability analysis of long-span roofing structures: the case-study of Porta Susa railway-station. Eng Struct. 2016;110:48-58.

[19] Bazzucchi F, Manuello A, Carpinteri A. Interaction between snapthrough and Eulerian instability in shallow structures. Int. J. Nonlin. Mech. 2016;88:11-20. 